\title{
EXISTENCE OF GLOBAL SOLUTION AND NONTRIVIAL STEADY STATES FOR A SYSTEM MODELING CHEMOTAXIS
}

\author{
ZHENBU ZHANG
}

Received 23 March 2006; Revised 18 September 2006; Accepted 19 September 2006

We consider a reaction-diffusion system modeling chemotaxis, which describes the situation of two species of bacteria competing for the same nutrient. We use Moser-Alikakos iteration to prove the global existence of the solution. We also study the existence of nontrivial steady state solutions and their stability.

Copyright (c) 2006 Zhenbu Zhang. This is an open access article distributed under the Creative Commons Attribution License, which permits unrestricted use, distribution, and reproduction in any medium, provided the original work is properly cited.

\section{Introduction}

Chemotaxis, the oriented movement of cells in response to ambient chemical gradients, is a prominent feature in the organization of many biological populations. Since the pioneer work of Keller and Segel [11] to propose mathematical models for chemotaxis, there has been great interest in modeling chemotaxis and in the mathematical analysis of systems like the Keller-Segel model. In this paper, motivated by the model in [15], we consider a revised model discussed in [16], that is,

$$
\begin{gathered}
\frac{\partial N}{\partial t}=\mu N_{x x}-R_{1}(N) b-R_{2}(N) B, \quad 0<x<1, t>0, \\
\frac{\partial b}{\partial t}=\left(d b_{x}-\alpha b S_{1}(N) N_{x}\right)_{x}+b\left(\rho_{1} R_{1}(N)-b-B\right), \quad 0<x<1, t>0, \\
\frac{\partial B}{\partial t}=\left(D B_{x}-\beta B S_{2}(N) N_{x}\right)_{x}+B\left(\rho_{2} R_{2}(N)-b-B\right), \quad 0<x<1, t>0, \\
N_{x}(0, t)=0, \quad N_{x}(1, t)=\gamma(1-N(1, t)), \quad t>0, \\
d b_{x}-\alpha b S_{1}(N) N_{x}=0, \quad x=0,1, t>0, \\
D B_{x}-\beta B S_{2}(N) N_{x}=0, \quad x=0,1, t>0, \\
N(x, 0)=N_{0}(x), \quad b(x, 0)=b_{0}(x), \quad B(x, 0)=B_{0}(x), \quad 0<x<1 .
\end{gathered}
$$


This is the situation of two species of bacteria competing for the same nutrient, where $N(x, t)$ is the concentration of the nutrient and $b(x, t), B(x, t)$ are the densities of two competing species of bacteria. $R_{i}(N), i=1,2$, are the consumption rates of the nutrient per cell. $-\mu N_{x},-d b_{x}$, and $-D B_{x}$ are the random fluxes of $N, b$, and $B$, respectively, while $\alpha b S_{1}(N) N_{x}$ and $\beta B S_{2}(N) N_{x}$ are the chemotactic fluxes of $b$ and $B$, where $\mu>0, d>0$, $D>0$ and $\alpha \geq 0, \beta \geq 0$. For definiteness, we assume that $d<D$. Functions $S_{i}(N), i=1,2$, the so-called sensitivity rates, are included to indicate that the sensitivity of cells to the nutrient may vary at different levels of nutrient concentration. When $\alpha=0, \beta=0$, and $\rho_{i}=1$, this model reduces to the model discussed in [16]. But the present model is not a trivial generalization of the model discussed in [16] because of the appearance of the chemotactic fluxes of $b$ and $B$. Due to the lack of monotone structure on the system, the main tool-the comparison principle-used in [16] does not work here. In [15], the authors considered a similar model and discussed the situations when there is no positive steady state. In this paper, we will give sufficient conditions that guarantee the existence of positive solutions. The method we use here to investigate the existence of steady states is different from that used in [15]. We also consider some special cases in which the sufficient conditions we will derive are not satisfied and the systems have no nontrivial steady states. The boundary conditions represent that the total fluxes of $b$ and $B$ at the boundary points $x=0$ and $x=1$ are zero. This is true for $N$ at $x=0$, but at $x=1$, $N$ is diffused into the medium. In the adjacent region, $N \equiv 1$, which must also be an upper bound for $N$ inside the medium, and therefore we are only interested in solutions with $0 \leq N \leq 1$. For this reason, we assume that $0 \leq N_{0} \leq 1$ throughout the paper.

From biological and technical considerations, we assume that

$$
R_{i}(0)=0, \quad R_{i}^{\prime}(N)>0, \quad S_{i}(N)>0 \quad \text { on }[0, \infty) .
$$

The assumptions about $R_{i}$ guarantee the nonnegativeness of $N, b$, and $B$ as long as the initial functions are nonnegative (see [13]). Therefore we will only consider nonnegative solutions of (1.1).

This paper is organized as follows. In Section 2, we will prove the global existence of solutions. In Section 3, we will study the existence of steady states and some special cases.

\section{Global existence}

By standard existence theory, for example, see $[3-5,12]$, it is not difficult to establish the local existence of the unique solution $(N(x, t), b(x, t), B(x, t))$ for $0 \leq t<T_{\max }$, where $T_{\max }$ is determined by $N_{0}, b_{0}$, and $B_{0}$. It is well known that local existence together with $L^{\infty}$ a priori bounds ensure the global existence of classical solutions. Therefore, to establish the global existence, we need only to establish a priori estimates for $\|N(\cdot, t)\|_{L^{\infty}},\|b(\cdot, t)\|_{L^{\infty}}$, and $\|B(\cdot, t)\|_{L^{\infty}}$. The boundedness of $\|N(\cdot, t)\|_{L^{\infty}}$ is trivial because we have $0 \leq N \leq 1$ (in fact, this can be proved directly by using comparison principle). Therefore we need only to establish the boundedness of $\|b(\cdot, t)\|_{L^{\infty}}$, and $\|B(\cdot, t)\|_{L^{\infty}}$. This is done by proving several lemmas. The following general imbedding result will be of use to us. 
Theorem 2.1 (see $[10,2]$ ). Assume that operator $\mathscr{A}$ is sectorial in $X=L^{p}(\Omega), 1<p<\infty$, where $\Omega$ is a bounded smooth domain in $\mathbb{R}^{n}$ and $X^{1}=D(\mathscr{A}) \hookrightarrow W^{m, p}(\Omega)$ (where $\hookrightarrow$ denotes topological as well as set inclusion) for some integer $m \geq 1$. Then for $0<\theta<1$,

(i) $X^{\theta} \hookrightarrow W^{l, q}(\Omega)$ if $l=0,1,2, \ldots, m-1,1 \leq q \leq+\infty$, where $\theta>l / m$ and $1 / q>1 / p-$ $(\theta m-l) / n$

(ii) $X^{\theta} \hookrightarrow C^{\nu}(\Omega)$ if $0 \leq v<\theta m-n / p$.

Lemma 2.2. If $1 \leq p<\infty$, and $f \geq 0, g \geq 0$, and $h \geq 0$, then

$$
(f+g+h)^{p} \leq 2^{2 p-2}\left(f^{p}+g^{p}+h^{p}\right) .
$$

Proof. It is well known that if $1 \leq p<\infty$, and $a \geq 0, b \geq 0$, then (e.g., see [1])

$$
(a+b)^{p} \leq 2^{p-1}\left(a^{p}+b^{p}\right) .
$$

Therefore,

$$
\begin{aligned}
(f+g+h)^{p} & \leq 2^{p-1}\left((f+g)^{p}+h^{p}\right) \leq 2^{p-1}\left(2^{p-1}\left(f^{p}+g^{p}\right)+h^{p}\right) \\
& \leq 2^{2 p-2}\left(f^{p}+g^{p}+h^{p}\right) .
\end{aligned}
$$

Lemma 2.3. There exist positive constants $c_{b}$ and $C_{B}$ such that for $0 \leq t<T_{\max }$,

$$
\begin{gathered}
\bar{b}(t)=\int_{0}^{1} b(x, t) d x \leq c_{b}, \\
\bar{B}(t)=\int_{0}^{1} B(x, t) d x \leq C_{B} .
\end{gathered}
$$

Proof. Let

$$
\Lambda(t)=\int_{0}^{1} b(x, t) d x+\int_{0}^{1} B(x, t) d x=\bar{b}(t)+\bar{B}(t) .
$$

Obviously, $\bar{b}(t) \geq 0$ and $\bar{B}(t) \geq 0$. Therefore, to prove the lemma, we need only to prove that there exists a constant $M>0$ such that for $0 \leq t<T_{\max }$,

$$
\Lambda(t) \leq M
$$

In fact, by adding the $b$-equation and $B$-equation in (1.1) and using the boundary conditions, we have

$$
\begin{aligned}
\Lambda^{\prime}(t) & =\int_{0}^{1}\left(\rho_{1} R_{1}(N) b(x, t)+\rho_{2} R_{2}(N) B(x, t)\right) d x-\int_{0}^{1}(b(x, t)+B(x, t))^{2} d x \\
& \leq R \int_{0}^{1}(b(x, t)+B(x, t)) d x-\left(\int_{0}^{1}(b(x, t)+B(x, t)) d x\right)^{2}=R \Lambda(t)-(\Lambda(t))^{2},
\end{aligned}
$$

where $R=\max \left\{\rho_{1} R_{1}(1), \rho_{2} R_{2}(1)\right\}$. This implies (2.6). 
Lemma 2.4. For any small $\tau>0$, there exists a positive constant $\delta$ depending on $R_{i}, S_{i}, b_{0}$, $B_{0}$, and $\|N(x, \tau)\|_{H^{2}(0,1)}$ such that

$$
\begin{aligned}
\max & \left\{\int_{0}^{1} b^{2}(x, t) d x, \int_{0}^{1} B^{2}(x, t) d x\right\} \\
& \leq 2\left(\int_{0}^{1} b^{2}(x, \tau) d x+\int_{0}^{1} B^{2}(x, \tau) d x\right)+\delta, \quad \tau \leq t<T_{\max } .
\end{aligned}
$$

Proof. For $1<p<2$, let $X=L^{p}(0,1)$. Operator $\mathscr{A}_{p}=-\mu\left(d^{2} / d x^{2}\right)$ with domain

$$
D\left(\mathscr{A}_{p}\right)=\left\{u \in W^{2, p}(0,1): u^{\prime}(0)=0=u^{\prime}(1)+\gamma u(1)\right\}
$$

is sectorial in $X$ and $\sigma\left(\mathscr{A}_{p}\right) \subset\left\{\mathbf{Z} \in \mathbb{R}: \mathbf{Z}>\lambda_{0}\right\}$ for a positive number $\lambda_{0}$ due to the symmetry of $\mathscr{A}_{p}$, where $\sigma\left(\mathscr{A}_{p}\right)$ is the spectrum of $\mathscr{A}_{p}$.

Since $\mathscr{A}_{p}$ is sectorial in $X$, the operator $-\mathscr{A}_{p}$ generates an analytic semigroup $\left\{\mathscr{T}_{p}(t)\right\}$ with $\left\|\mathscr{T}_{p}(t)\right\|_{X} \leq k e^{-\lambda_{0} t}$ for $t \geq 0$, for a positive constant $k$.

By Theorem 2.1, we know that, for $1 \geq \theta>1 / 4+1 / 2 p$, fractional space $X^{\theta} \hookrightarrow H^{1}(0,1)$. By Theorem 1.4.3 in [10], there exists a constant $k_{\theta}<\infty$ such that

$$
\left\|\mathscr{T}_{p}(t)\right\|_{X^{\theta}} \leq k_{\theta} t^{-\theta} e^{-\lambda_{0} t} .
$$

Let $u=1-N$, then $u$ satisfies

$$
\begin{gathered}
u_{t}=\mu u_{x x}+R_{1}(N) b+R_{2}(N) B, \quad 0<x<1, t>0, \\
u_{x}(0, t)=0, \quad u_{x}(1, t)+\gamma u(1, t)=0, \quad t>0, \\
u(x, \tau)=1-N(x, \tau), \quad 0<x<1 .
\end{gathered}
$$

Therefore $u \in D\left(\mathscr{A}_{p}\right)$ and for $\tau \leq t<T_{\max }$,

$$
\begin{aligned}
u(\cdot, t)= & \mathscr{T}_{p}(t-\tau)(1-N(\cdot, \tau)) \\
& +\int_{\tau}^{t} \mathscr{T}_{p}(t-\xi)\left(R_{1}(N(\cdot, \xi)) b(\cdot, \xi)+R_{2}(N(\cdot, \xi)) B(\cdot, \xi)\right) d \xi .
\end{aligned}
$$

Now for $\tau \leq t<T_{\max }$, we have

$$
\begin{aligned}
\left\|N_{x}(\cdot, t)\right\|_{L^{2}(0,1)} \leq & \|u(\cdot, t)\|_{H^{1}(0,1)} \leq C\|u(\cdot, t)\|_{X^{\theta}} \leq C\left\|\mathscr{T}_{p}(t-\tau)(1-N(\cdot, \tau))\right\|_{X^{\theta}} \\
& +C \int_{\tau}^{t}\left\|\mathcal{T}_{p}(t-\xi)\left(R_{1}(N(\cdot, \xi)) b(\cdot, \xi)+R_{2}(N(\cdot, \xi)) B(\cdot, \xi)\right)\right\|_{X^{\theta}} d \xi \\
\leq & C\left\|\mathcal{T}_{p}(t-\tau)\right\|_{X}\|1-N(\cdot, \tau)\|_{X^{\theta}} \\
& +C \int_{\tau}^{t}\left\|\mathcal{T}_{p}(t-\xi)\right\|_{X^{\theta}}\left\|R_{1}(N(\cdot, \xi)) b(\cdot, \xi)+R_{2}(N(\cdot, \xi)) B(\cdot, \xi)\right\|_{X} d \xi \\
\leq & K e^{-\lambda_{0}(t-\tau)}\|1-N(\cdot, \tau)\|_{X^{\theta}}+C R_{1}(1) \int_{\tau}^{t} k_{\theta}(t-\xi)^{-\theta} e^{-\lambda_{0}(t-\xi)}\|b(\cdot, \xi)\|_{X} d \xi \\
& +C R_{2}(1) \int_{\tau}^{t} k_{\theta}(t-\xi)^{-\theta} e^{-\lambda_{0}(t-\xi)}\|B(\cdot, \xi)\|_{X} d \xi .
\end{aligned}
$$


Let

$$
\begin{aligned}
& \omega(t)=\max _{\tau \leq \xi<t} \int_{0}^{1} b^{2}(x, \xi) d x, \\
& \Gamma(t)=\max _{\tau \leq \xi<t} \int_{0}^{1} B^{2}(x, \xi) d x,
\end{aligned}
$$

then it is easily seen that $\omega(t)$ and $\Gamma(t)$ are nondecreasing functions of $t$. By Hölder's inequality

$$
\begin{gathered}
\|b(\cdot, \xi)\|_{X} \leq \bar{b}^{(2-p) / p}(t)\left(\int_{0}^{1} b^{2}(x, \xi) d x\right)^{(p-1) / p} \leq \bar{b}^{(2-p) / p}(t)(\omega(t))^{(p-1) / p}, \\
\|B(\cdot, \xi)\|_{X} \leq \bar{B}^{(2-p) / p}(t)(\Gamma(t))^{(p-1) / p}
\end{gathered}
$$

where $\bar{b}(t)$ and $\bar{B}(t)$ are defined in Lemma 2.3. Therefore, from (2.13) and Lemma 2.3, we know that there exist constants $k_{1}$ depending on $\|N(x, \tau)\|_{H^{2}(0,1)}, k_{2}$, and $k_{3}$ depending on $R_{i}$ such that

$$
\left\|N_{x}(\cdot, t)\right\|_{L^{2}(0,1)} \leq k_{1}+k_{2}(\omega(t))^{(p-1) / p}+k_{3}(\Gamma(t))^{(p-1) / p} .
$$

Now multiplying $b(x, t)$ to the $b$-equation in $(1.1)$ and integrating by parts on $[0,1]$, we obtain that for $\tau \leq t<T_{\max }$,

$$
\begin{aligned}
\frac{1}{2} \frac{d}{d t} \int_{0}^{1} b^{2}(x, t) d x \leq & -\int_{0}^{1}\left(d b_{x}-\alpha b S_{1}(N) N_{x}\right) b_{x} d x+\int_{0}^{1} \rho_{1} R_{1}(N) b^{2}(x, t) d x \\
\leq & -d \int_{0}^{1} b_{x}^{2} d x+\alpha \int_{0}^{1} S_{1}(N) N_{x} b b_{x} d x+\rho_{1} R_{1}(1) \int_{0}^{1} b^{2}(x, t) d x \\
\leq & -d \int_{0}^{1} b_{x}^{2} d x+c_{1}\|b(\cdot, t)\|_{L^{\infty}(0,1)}\left(\int_{0}^{1} N_{x}^{2} d x\right)^{1 / 2}\left(\int_{0}^{1} b_{x}^{2} d x\right)^{1 / 2} \\
& +\rho_{1} R_{1}(1) \int_{0}^{1} b^{2}(x, t) d x .
\end{aligned}
$$

From this and the inequality (see [14])

$$
\|b(\cdot, t)\|_{L^{\infty}(0,1)} \leq c\left(\bar{b}(t)+\bar{b}^{1 / 3}(t)\left(\int_{0}^{1} b_{x}^{2}(x, t) d x\right)^{1 / 3}\right)
$$


we have

$$
\begin{aligned}
& \frac{1}{2} \frac{d}{d t} \int_{0}^{1} b^{2}(x, t) d x \\
& \leq-d \int_{0}^{1} b_{x}^{2} d x+c_{2}\left(\bar{b}(t)+\bar{b}^{1 / 3}(t)\left(\int_{0}^{1} b_{x}^{2} d x\right)^{1 / 3}\right)\left(\int_{0}^{1} N_{x}^{2} d x\right)^{1 / 2}\left(\int_{0}^{1} b_{x}^{2} d x\right)^{1 / 2} \\
& \quad+\rho_{1} R_{1}(1) \int_{0}^{1} b^{2}(x, t) d x \\
& =-d \int_{0}^{1} b_{x}^{2} d x+c_{2} \bar{b}(t)\left(\int_{0}^{1} N_{x}^{2} d x\right)^{1 / 2}\left(\int_{0}^{1} b_{x}^{2} d x\right)^{1 / 2} \\
& \quad+c_{2} \bar{b}^{1 / 3}(t)\left(\int_{0}^{1} N_{x}^{2} d x\right)^{1 / 2}\left(\int_{0}^{1} b_{x}^{2} d x\right)^{5 / 6}+\rho_{1} R_{1}(1) \int_{0}^{1} b^{2}(x, t) d x
\end{aligned}
$$

From Young's inequality

$$
X Y \leq \eta X^{p}+c(\eta) Y^{q}
$$

(where $1 / p+1 / q=1, c(\eta)=(\eta p)^{-q / p / q)}$ with $p=q=2, \eta=d / 4$ and $p=6 / 5, q=6$, $\eta=d / 4$, respectively, we have

$$
\begin{gathered}
c_{2} \bar{b}(t)\left(\int_{0}^{1} N_{x}^{2} d x\right)^{1 / 2}\left(\int_{0}^{1} b_{x}^{2} d x\right)^{1 / 2} \leq \frac{d}{4} \int_{0}^{1} b_{x}^{2} d x+\frac{1}{d} c_{2}^{2} \bar{b}^{2}(t) \int_{0}^{1} N_{x}^{2} d x, \\
c_{2} \bar{b}^{1 / 3}(t)\left(\int_{0}^{1} N_{x}^{2} d x\right)^{1 / 2}\left(\int_{0}^{1} b_{x}^{2} d x\right)^{5 / 6} \leq \frac{d}{4} \int_{0}^{1} b_{x}^{2} d x+c_{3} \bar{b}^{2}(t)\left(\int_{0}^{1} N_{x}^{2} d x\right)^{3} .
\end{gathered}
$$

Using (2.21) in (2.19), we obtain

$$
\begin{aligned}
\frac{1}{2} \frac{d}{d t} \int_{0}^{1} b^{2}(x, t) d x \leq & -d \int_{0}^{1} b_{x}^{2} d x+\frac{d}{4} \int_{0}^{1} b_{x}^{2} d x+\frac{1}{d} c_{2}^{2} \bar{b}^{2}(t) \int_{0}^{1} N_{x}^{2} d x \\
& +\frac{d}{4} \int_{0}^{1} b_{x}^{2} d x+c_{3} \bar{b}^{2}(t)\left(\int_{0}^{1} N_{x}^{2} d x\right)^{3}+\rho_{1} R_{1}(1) \int_{0}^{1} b^{2}(x, t) d x \\
= & -\frac{d}{2} \int_{0}^{1} b_{x}^{2} d x+\frac{1}{d} c_{2}^{2} \bar{b}^{2}(t) \int_{0}^{1} N_{x}^{2} d x+c_{3} \bar{b}^{2}(t)\left(\int_{0}^{1} N_{x}^{2} d x\right)^{3} \\
& +\rho_{1} R_{1}(1) \int_{0}^{1} b^{2}(x, t) d x \\
\leq & -\frac{d}{2} \int_{0}^{1} b_{x}^{2} d x+c_{4} \bar{b}^{2}(t)+c_{5} \bar{b}^{2}(t)\left(\int_{0}^{1} N_{x}^{2} d x\right)^{3}+\rho_{1} R_{1}(1) \int_{0}^{1} b^{2}(x, t) d x .
\end{aligned}
$$

For any $\epsilon>0$, from the following inequality (see [14]),

$$
\int_{0}^{1} b^{2}(x, t) d x \leq \epsilon \int_{0}^{1} b_{x}^{2} d x+\left(c \epsilon^{-1 / 2}+1\right) \bar{b}^{2}(t)
$$


we have

$$
\int_{0}^{1} b_{x}^{2} d x \geq \frac{1}{\epsilon}\left(\int_{0}^{1} b^{2}(x, t) d x-\left(c \epsilon^{-1 / 2}+1\right) \bar{b}^{2}(t)\right) .
$$

Therefore,

$$
\begin{aligned}
\frac{1}{2} \frac{d}{d t} \int_{0}^{1} b^{2}(x, t) d x \leq & -\frac{d}{2 \epsilon}\left(\int_{0}^{1} b^{2}(x, t) d x-\left(c \epsilon^{-1 / 2}+1\right) \bar{b}^{2}(t)\right) \\
& +c_{4} \bar{b}^{2}(t)+c_{5} \bar{b}^{2}(t)\left(\int_{0}^{1} N_{x}^{2} d x\right)^{3}+\rho_{1} R_{1}(1) \int_{0}^{1} b^{2}(x, t) d x \\
\leq & \left(\rho_{1} R_{1}(1)-\frac{d}{2 \epsilon}\right) \int_{0}^{1} b^{2}(x, t) d x+c_{6} \bar{b}^{2}(t)+c_{5} \bar{b}^{2}(t)\left(\int_{0}^{1} N_{x}^{2} d x\right)^{3}
\end{aligned}
$$

By taking $\epsilon=d /\left(3 \rho_{1} R_{1}(1)\right)$, we obtain

$$
\frac{1}{2} \frac{d}{d t} \int_{0}^{1} b^{2}(x, t) d x \leq-\frac{\rho_{1} R_{1}(1)}{2} \int_{0}^{1} b^{2}(x, t) d x+c_{6} \bar{b}^{2}(t)+c_{5} \bar{b}^{2}(t)\left(\int_{0}^{1} N_{x}^{2} d x\right)^{3}
$$

Then, in view of (2.16) and Lemma 2.2, we have

$$
\begin{aligned}
\frac{1}{2} \frac{d}{d t} \int_{0}^{1} b^{2}(x, t) d x \leq & -\frac{\rho_{1} R_{1}(1)}{2} \int_{0}^{1} b^{2}(x, t) d x \\
& +c_{6} \bar{b}^{2}(t)+c_{5} \bar{b}^{2}(t)\left(k_{1}+k_{2}(\omega(t))^{(p-1) / p}+k_{3}(\Gamma(t))^{(p-1) / p}\right)^{6} \\
\leq & -\frac{\rho_{1} R_{1}(1)}{2} \int_{0}^{1} b^{2}(x, t) d x+c_{7} \bar{b}^{2}(t)+c_{8} \bar{b}^{2}(t)(\omega(t))^{6(p-1) / p} \\
& +c_{9} \bar{b}^{2}(t)(\Gamma(t))^{6(p-1) / p} .
\end{aligned}
$$

From this, the monotonicity of $\omega(t)$ and $\Gamma(t)$ and Lemma 2.3, it follows that for $\tau \leq t<$ $T_{\max }$,

$$
\int_{0}^{1} b^{2}(x, t) d x \leq \int_{0}^{1} b^{2}(x, \tau) d x+c_{10}\left(1+(\omega(t))^{6(p-1) / p}+(\Gamma(t))^{6(p-1) / p}\right) .
$$

Thus

$$
\omega(t) \leq \int_{0}^{1} b^{2}(x, \tau) d x+c_{10}\left(1+(\omega(t))^{6(p-1) / p}+(\Gamma(t))^{6(p-1) / p}\right) .
$$


Similarly, we have

$$
\Gamma(t) \leq \int_{0}^{1} B^{2}(x, \tau) d x+c_{11}\left(1+(\omega(t))^{6(p-1) / p}+(\Gamma(t))^{6(p-1) / p}\right) .
$$

Therefore,

$$
\begin{aligned}
\omega(t)+\Gamma(t) & \leq \int_{0}^{1} b^{2}(x, \tau) d x+\int_{0}^{1} B^{2}(x, \tau) d x+c_{12}\left(1+(\omega(t))^{6(p-1) / p}+(\Gamma(t))^{6(p-1) / p}\right) \\
& \left.\leq \int_{0}^{1} b^{2}(x, \tau) d x+\int_{0}^{1} B^{2}(x, \tau) d x+c_{12}+c_{13}(\omega(t))+\Gamma(t)\right)^{6(p-1) / p} .
\end{aligned}
$$

Now we take $6 / 5>p>1$, then $6(p-1) / p<1$. Therefore there exists a positive constant $\delta$ depending on $R_{i}, S_{i}, \rho_{i}, b_{0}, B_{0}$, and $\|N(x, \tau)\|_{H^{2}(0,1)}$ such that

$$
\omega(t)+\Gamma(t) \leq 2\left(\int_{0}^{1} b^{2}(x, \tau) d x+\int_{0}^{1} B^{2}(x, \tau) d x\right)+\delta, \quad \tau \leq t<T_{\max }
$$

From this we know that the lemma is true.

LEMma 2.5. For any small $\tau>0$, there exists a positive constant $L=L\left(T_{\max }\right)$ such that

$$
\left\|N_{x}(\cdot, t)\right\|_{L^{\infty}(0,1)} \leq L, \quad \tau \leq t<T_{\max }
$$

Proof. Let $X=L^{2}(0,1)$. Operator $\mathscr{A}_{2}=-\mu\left(d^{2} / d x^{2}\right)$ with domain

$$
D\left(\mathscr{A}_{2}\right)=\left\{u \in H^{2}(0,1): u^{\prime}(0)=0=u^{\prime}(1)+\gamma u(1)\right\}
$$

is sectorial in $X$ and $\sigma\left(\mathscr{A}_{2}\right) \subset\left\{\mathbf{Z} \in \mathbb{R}: \mathbf{Z}>\lambda_{0}\right\}$ for a positive number $\lambda_{0}$ due to the symmetry of $\mathscr{A}_{2}$, where $\sigma\left(\mathscr{A}_{2}\right)$ is the spectrum of $\mathscr{A}_{2}$.

Since $\mathscr{A}_{2}$ is sectorial in $X$, the operator $-\mathscr{A}_{2}$ generates an analytic semigroup $\left\{\mathscr{T}_{2}(t)\right\}$ with $\left\|\mathscr{T}_{2}(t)\right\|_{X} \leq k e^{-\lambda_{0} t}$, for some constant $k$, for $t \geq 0$.

By Theorem 2.1, we know that, for $1>\theta>1 / 4$, fractional space $X^{\theta} \hookrightarrow C^{\nu}[0,1]$ for $\nu \in$ $(0,2 \theta-1 / 2)$. In particular, we take $\theta>3 / 4$, then $\nu$ can be taken to be 1 and $X^{\theta} \hookrightarrow C^{1}[0,1]$. 
Therefore, from (2.12) with $p=2$, we have, for $\tau \leq t<T_{\max }$,

$$
\begin{aligned}
\left\|N_{x}(\cdot, t)\right\|_{C[0,1]} \leq & \|u(\cdot, t)\|_{C^{1}[0,1]} \leq\|u(\cdot, t)\|_{X^{\theta}} \leq\left\|\mathscr{T}_{2}(t-\tau)(1-N(\cdot, \tau))\right\|_{X^{\theta}} \\
& +\int_{\tau}^{t}\left\|\mathscr{T}_{2}(t-\xi)\left(R_{1}(N(\cdot, \xi)) b(\cdot, \xi)+R_{2}(N(\cdot, \xi)) B(\cdot, \xi)\right)\right\|_{X^{\theta}} d \xi \\
\leq & \left\|\mathscr{T}_{2}(t-\tau)\right\|_{X}\|1-N(\cdot, \tau)\|_{X^{\theta}} \\
& +\int_{\tau}^{t}\left\|\mathscr{T}_{2}(t-\xi)\right\|_{X^{\theta}}\left\|\left(R_{1}(N(\cdot, \xi)) b(\cdot, \xi)+R_{2}(N(\cdot, \xi)) B(\cdot, \xi)\right)\right\|_{X} d \xi \\
\leq & k e^{-\lambda_{0}(t-\tau)}\|1-N(\cdot, \tau)\|_{X^{\theta}}+R \int_{\tau}^{t} k_{\theta}(t-\xi)^{-\theta} e^{-\lambda_{0}(t-\xi)}\|b(\cdot, \xi)+B(\cdot, \xi)\|_{X} d \xi \\
\leq & k e^{-\lambda_{0}(t-\tau)}\|1-N(\cdot, \tau)\|_{X^{\theta}} \\
& +R \int_{\tau}^{t} k_{\theta}(t-\xi)^{-\theta} e^{-\lambda_{0}(t-\xi)}\left(\|b(\cdot, \xi)\|_{X}+\|B(\cdot, \xi)\|_{X}\right) d \xi \\
\leq & k e^{-\lambda_{0}(t-\tau)}\|1-N(\cdot, \tau)\|_{X^{\theta}} \\
& +R \int_{\tau}^{t} 2\left(2\left(\int_{0}^{1} b^{2}(x, \tau) d x+\int_{0}^{1} B^{2}(x, \tau) d x\right)+\delta\right)^{1 / 2} k_{\theta}(t-\xi)^{-\theta} e^{-\lambda_{0}(t-\xi)} d \xi \\
= & k e^{-\lambda_{0}(t-\tau)}\|1-N(\cdot, \tau)\|_{X^{\theta}} \\
& +2 R\left(2\left(\int_{0}^{1} b^{2}(x, \tau) d x+\int_{0}^{1} B^{2}(x, \tau) d x\right)+\delta\right)^{1 / 2} \int_{\tau}^{t} k_{\theta}(t-\xi)^{-\theta} e^{-\lambda_{0}(t-\xi)} d \xi \\
& 2 R
\end{aligned}
$$

where $R=\max \left\{R_{1}(1), R_{2}(1)\right\}$. This completes the proof.

LEMMA 2.6. There exists a positive constant $M>0$ such that for $\tau \leq t<T_{\max }$,

$$
\max \left\{\|b(\cdot, t)\|_{L^{\infty}},\|B(\cdot, t)\|_{L^{\infty}}\right\} \leq M .
$$

The proof is similar to that of Lemma 4.7 in [14] and therefore is omitted.

Thus we have the following global existence and boundedness theorem.

Theorem 2.7 (global existence and boundedness). For any $N_{0}, b_{0}$, and $B_{0} \in H^{1}(0,1)$ satisfying $N_{0}(x)>0, b_{0}(x)>0$, and $B_{0}(x)>0$ on $[0,1],(1.1)$ has a unique positive global solution $(N, b, B)$ such that

(i) $(N(x, t), b(x, t), B(x, t)) \in C\left([0, \infty), H^{1}(0,1) \times H^{1}(0,1) \times H^{1}(0,1)\right)$,

$$
(N, b, B) \in C_{\mathrm{loc}}^{2+2 \epsilon, 1+\epsilon}([0,1] \times(0, \infty)) ;
$$

(ii) $N>0, b>0$, and $B>0$ are bounded on $[0,1] \times[0, \infty)$.

\section{Existence of steady states}

In this section, we study the existence of steady states of (1.1). Basically, we study the existence of nontrivial steady state solutions of (1.1) in the framework of [9]. But in [9], the 
author made several assumptions about the reaction terms. Unfortunately, in our model the reaction functions do not satisfy all these assumptions. This fact causes difficulties in using the theory developed in [9]. Therefore, we must do some careful and technical analysis for our model. The steady states of (1.1) satisfy

$$
\begin{gathered}
\mu N^{\prime \prime}-R_{1}(N) b-R_{2}(N) B=0, \quad 0<x<1, \\
\left(d b^{\prime}-\alpha b S_{1}(N) N^{\prime}\right)^{\prime}+b\left(\rho_{1} R_{1}(N)-b-B\right)=0, \quad 0<x<1, \\
\left(D B^{\prime}-\beta B S_{2}(N) N^{\prime}\right)^{\prime}+B\left(\rho_{2} R_{2}(N)-b-B\right)=0, \quad 0<x<1, \\
N^{\prime}(0)=0, \quad N^{\prime}(1)=\gamma(1-N(1)), \\
d b^{\prime}-\alpha b S_{1}(N) N^{\prime}=0 \quad \text { at } x=0,1, \\
D B^{\prime}-\beta B S_{2}(N) N^{\prime}=0 \quad \text { at } x=0,1 .
\end{gathered}
$$

Obviously, $(1,0,0)$ is a solution of (3.1), that is, it is a steady state of (1.1). For this, we have the following theorem.

Theorem 3.1. The trivial steady state solution $(N, b, B)=(1,0,0)$ is unstable.

Proof. To prove this theorem, we use the definition of instability (e.g., see [6]). That is, if $O_{\epsilon}$ is a neighborhood of $(1,0,0)$ consisting of $(N, b, B)$ such that

$$
\|1-N\|_{L^{\infty}}+\|b\|_{L^{\infty}}+\|B\|_{L^{\infty}}<\epsilon,
$$

we can show that for a small $\epsilon>0$, the solution $(N(x, t), b(x, t), B(x, t))$ always leaves $O_{\epsilon}$ in finite time no matter how close the initial values $\left(N_{0}, b_{0}, B_{0}\right)$ are to $(1,0,0)$. In fact, for $\epsilon>0$ small, we have

$$
\rho_{1} R_{1}(N)-b-B \geq \frac{\rho_{1} R_{1}(1)}{2}>0, \quad x \in[0,1], t>0 .
$$

Then by integrating the $b$-equation in (1.1), we have

$$
\frac{d}{d t} \int_{0}^{1} b(x, t) d x \geq \frac{\rho_{1} R_{1}(1)}{2} \int_{0}^{1} b(x, t) d x, \quad t>0 .
$$

It follows that

$$
\int_{0}^{1} b(x, t) d x \geq e^{\left(\rho_{1} R_{1}(1) / 2\right) t} \int_{0}^{1} b_{0}(x) d x \longrightarrow \infty \quad \text { as } t \longrightarrow \infty .
$$

This implies that $(N(x, t), b(x, t), B(x, t))$ must leave $O_{\epsilon}$ in finite time.

LEMmA 3.2. If $(N(x), b(x), B(x))$ is a solution of (3.1) such that at least one of the functions $b(x)$ and $B(x)$ is positive, then

$$
0<N^{\prime}(x)<\gamma \quad \text { for } 0<x \leq 1, \quad 0<N(x)<1 \quad \text { for } 0<x \leq 1,
$$

and there exists a positive constant $K_{1}$ such that

$$
\max \left\{\|b\|_{L^{1}},\|B\|_{L^{1}}\right\} \leq K_{1} .
$$


Also

(i) $0 \leq b(x) \leq \rho_{1} R_{1}(1) e^{(\alpha / d) \int_{0}^{1} S_{1}(y) d y}$, and $0 \leq B(x) \leq \rho_{2} R_{2}(1) e^{(\beta / D) \int_{0}^{1} S_{2}(y) d y}$,

(ii) there exists a positive constant $K_{2}$ such that

$$
\max \left\{\left|b^{\prime}(x)\right|,\left|B^{\prime}(x)\right|\right\} \leq K_{2}
$$

Proof. From the assumptions and the first equation of $(3.1)$, we have $N^{\prime \prime}(x)>0$. Therefore $N^{\prime}(x)$ is increasing. From $N^{\prime}(0)=0$, we know that $N^{\prime}(x)>0$ for $0<x \leq 1$. In particular, we have $N^{\prime}(1)>0$. Then the $N$-boundary condition at $x=1$ implies that $N^{\prime}(1)<\gamma$. Since $N^{\prime}(x)$ is increasing, we must have $N^{\prime}(x)<\gamma$ for $0 \leq x \leq 1$. Also, from the $N$ boundary condition at $x=1$, we have $N(1)<1$. Observe that $N(x)$ is also increasing, hence for $0 \leq x \leq 1, N(x)<1$. By the comparison principle and the condition $R_{i}(0)=0$, we have $N(x)>0$ for $x>0$.

Integrating the $b$-equation in (3.1) from 0 to 1 and using the boundary conditions, we have

$$
\int_{0}^{1} b\left(\rho_{1} R_{1}(N)-b-B\right) d x=0
$$

Therefore,

$$
\int_{0}^{1} b \rho_{1} R_{1}(N) d x=\int_{0}^{1} b(b+B) d x=\int_{0}^{1} b^{2} d x+\int_{0}^{1} b B d x
$$

Similarly,

$$
\int_{0}^{1} B \rho_{2} R_{2}(N) d x=\int_{0}^{1} B(b+B) d x=\int_{0}^{1} B^{2} d x+\int_{0}^{1} b B d x .
$$

Adding (3.10) and (3.11), we obtain

$$
\int_{0}^{1}\left(\rho_{1} R_{1}(N) b+\rho_{2} R_{2}(N) B\right) d x=\int_{0}^{1}(b+B)^{2} d x \geq\left(\int_{0}^{1}(b+B) d x\right)^{2} .
$$

It follows that

$$
\left(\int_{0}^{1}(b+B) d x\right)^{2} \leq \int_{0}^{1}\left(\rho_{1} R_{1}(N) b+\rho_{2} R_{2}(N) B\right) d x \leq \bar{R} \int_{0}^{1}(b+B) d x,
$$

where $\bar{R}=\max \left\{\rho_{1} R_{1}(1), \rho_{2} R_{2}(1)\right\}$. This implies that there is a constant $K$ such that

$$
\int_{0}^{1}(b+B) d x \leq K
$$

In turn, this implies that (3.7) is true.

Let $G_{1}(\tau)=\int_{0}^{\tau} S_{1}(y) d y \geq 0, G_{2}(\tau)=\int_{0}^{\tau} S_{2}(y) d y \geq 0$ and $z=e^{-(\alpha / d) G_{1}(N)} b, Z=$ $e^{-(\beta / D) G_{2}(N)} B$, then we have

$$
b^{\prime}-\frac{\alpha}{d} S_{1}(N) N^{\prime} b=e^{(\alpha / d) G_{1}(N)} z^{\prime},
$$


12 A system modeling chemotaxis

and $z$ satisfies

$$
\begin{gathered}
\left(e^{(\alpha / d) G_{1}(N)} z^{\prime}\right)^{\prime}+d^{-1} e^{(\alpha / d) G_{1}(N)} z\left(\rho_{1} R_{1}(N)-e^{(\alpha / d) G_{1}(N)} z-e^{(\beta / D) G_{2}(N)} Z\right)=0, \quad 0<x<1, \\
z^{\prime}(0)=z^{\prime}(1)=0 .
\end{gathered}
$$

Assume that $z(x)$ has its maximum at $x_{1}$. Then $z^{\prime}\left(x_{1}\right)=0$ and $z^{\prime \prime}\left(x_{1}\right) \leq 0$. From the above equation, we have, at $x_{1}$,

$$
\rho_{1} R_{1}(N)-e^{(\alpha / d) G_{1}(N)} z-e^{(\beta / D) G_{2}(N)} Z \geq 0 .
$$

Therefore,

$$
z\left(x_{1}\right) \leq \rho_{1} R_{1}(N) e^{-(\alpha / d) G_{1}(N)} \leq \rho_{1} R_{1}(1) .
$$

This implies

$$
z(x) \leq \rho_{1} R_{1}(1)
$$

Thus we have

$$
b(x) \leq \rho_{1} R_{1}(1) e^{(\alpha / d) G_{1}(1)}=\rho_{1} R_{1}(1) e^{(\alpha / d) \int_{0}^{1} S_{1}(y) d y} .
$$

Similarly,

$$
B(x) \leq \rho_{2} R_{2}(1) e^{(\beta / D) \int_{0}^{1} S_{2}(y) d y} .
$$

Integrating the $b$-equation from 0 to $x$ and using the $b$-boundary condition at $x=0$, we have

$$
\begin{aligned}
d b^{\prime}(x)= & \alpha b S_{1}(N) N^{\prime}+\int_{0}^{x} b\left(b+B-\rho_{1} R_{1}(N)\right) d s \\
\leq & \alpha b S_{1}(1) \gamma+\int_{0}^{x} b(b+B) d s \leq \alpha b S_{1}(1) \gamma+\int_{0}^{1} b(b+B) d s \\
\leq & \alpha \rho_{1} R_{1}(1) e^{(\alpha / d) \int_{0}^{1} S_{1}(y) d y} S_{1}(1) \gamma \\
& +\int_{0}^{1} \rho_{1} R_{1}(1) e^{(\alpha / d) \int_{0}^{1} S_{1}(y) d y}\left(\rho_{1} R_{1}(1) e^{(\alpha / d) \int_{0}^{1} S_{1}(y) d y}+\rho_{2} R_{2}(1) e^{(\beta / D) \int_{0}^{1} S_{2}(y) d y}\right) d s \\
= & \alpha \rho_{1} R_{1}(1) e^{(\alpha / d) \int_{0}^{1} S_{1}(y) d y} S_{1}(1) \gamma \\
& +\rho_{1} R_{1}(1) e^{(\alpha / d) \int_{0}^{1} S_{1}(y) d y}\left(\rho_{1} R_{1}(1) e^{(\alpha / d) \int_{0}^{1} S_{1}(y) d y}+\rho_{2} R_{2}(1) e^{(\beta / D) \int_{0}^{1} S_{2}(y) d y}\right) \\
\leq & \rho_{1} R_{1}(1) e^{(\alpha / d) \int_{0}^{1} S_{1}(y) d y}\left(\alpha S_{1}(1) \gamma+R\left(e^{(\alpha / d) \int_{0}^{1} S_{1}(y) d y}+e^{(\beta / D) \int_{0}^{1} S_{2}(y) d y}\right)\right) .
\end{aligned}
$$

Therefore,

$$
b^{\prime}(x) \leq d^{-1}\left\{\rho_{1} R_{1}(1) e^{(\alpha / d) \int_{0}^{1} S_{1}(y) d y}\left(\alpha S_{1}(1) y+R\left(e^{(\alpha / d) \int_{0}^{1} S_{1}(y) d y}+e^{(\beta / D) \int_{0}^{1} S_{2}(y) d y}\right)\right)\right\} .
$$


We also have

$$
\begin{aligned}
d b^{\prime}(x) & =\alpha b S_{1}(N) N^{\prime}+\int_{0}^{x} b\left(b+B-\rho_{1} R_{1}(N)\right) d s \geq \int_{0}^{x} b\left(-\rho_{1} R_{1}(N)\right) d s \\
& \geq \int_{0}^{x} \rho_{1} R_{1}(1) e^{(\alpha / d) \int_{0}^{1} S_{1}(y) d y}\left(-\rho_{1} R_{1}(1)\right) d s \geq-\left(\rho_{1} R_{1}(1)\right)^{2} e^{(\alpha / d) \int_{0}^{1} S_{1}(y) d y} .
\end{aligned}
$$

Now, let

$$
M_{1}=d^{-1}\left\{\rho_{1} R_{1}(1) e^{(\alpha / d) \int_{0}^{1} S_{1}(y) d y}\left(\alpha S_{1}(1) \gamma+R\left(e^{(\alpha / d) \int_{0}^{1} S_{1}(y) d y}+e^{(\beta / D) \int_{0}^{1} S_{2}(y) d y}\right)\right)\right\},
$$

then

$$
\left|b^{\prime}(x)\right| \leq M_{1}
$$

We can prove a similar estimate for $\left|B^{\prime}(x)\right|$. Therefore (3.8) is true.

COROLlARY 3.3. For any $v \in(0,1)$, there is a positive constant $K$ such that for any nontrivial solution $(N, b, B)$ of (3.1),

$$
\max \left\{\|N\|_{C^{v}([0,1])},\|b\|_{C^{\nu}([0,1])},\|B\|_{C^{v}([0,1])}\right\} \leq K .
$$

What we are interested in is whether (3.1) has any nontrivial solutions. The case $N \not \equiv 0$ is excluded by the boundary conditions. Therefore we need only to consider the possibilities of the existence of following two types of solutions:

(i) semitrivial solutions: $(N, b, 0),(N, 0, B)$;

(ii) positive solutions: $(N, b, B)$,

where the components $N>0, b>0, B>0$. In what follows, we use the theory of fixed point index on cones in a Banach space to study the existence of solutions of these types. First we study the existence of semitrivial solutions.

3.1. Existence of semitrivial solutions. From the symmetry of $b$ and $B$, we need only to study the existence of solutions of the form $(N, b, 0)$. For the convenience of notations, we write $N$ and $b$ as $u_{0}$ and $u_{1}$, respectively, omit the subscripts of $R_{1}, S_{1}$, and $\rho_{1}$, and consider the system

$$
\begin{gathered}
\mu u_{0}^{\prime \prime}-R\left(u_{0}\right) u_{1}=0, \quad 0<x<1, \\
\left(d u_{1}^{\prime}-\alpha u_{1} S\left(u_{0}\right) u_{0}^{\prime}\right)^{\prime}+u_{1}\left(\rho R\left(u_{0}\right)-u_{1}\right)=0, \quad 0<x<1, \\
u_{0}^{\prime}(0)=0, \quad u_{0}^{\prime}(1)=\gamma\left(1-u_{0}(1)\right), \\
d u_{1}^{\prime}-\alpha u_{1} S\left(u_{0}\right) u_{0}^{\prime}=0 \quad \text { at } x=0,1 .
\end{gathered}
$$

For $v \in(0,1)$, let

$$
\begin{gathered}
E_{i}=\left\{u \in C^{\nu}([0,1])\right\}, \quad i=0,1, \\
C_{i}=\left\{u \in C^{\nu}([0,1]): u \geq 0 \text { on }[0,1]\right\}, \quad i=0,1, \\
E=E_{0} \oplus E_{1}, \quad C=C_{0} \oplus C_{1},
\end{gathered}
$$


then $E$ is an ordered Banach space with positive cone $C$. For $V=\left(v_{0}, v_{1}\right) \in C$, let $u_{0}=$ $A_{0}(V)$ be the solution of

$$
\begin{gathered}
u_{0}^{\prime \prime}=\mu^{-1} R\left(u_{0}\right) v_{1}, \quad 0<x<1, \\
u_{0}^{\prime}(0)=0, \quad u_{0}^{\prime}(1)+\gamma u_{0}(1)=\gamma .
\end{gathered}
$$

With $u_{0}=u_{0}\left(v_{0}, v_{1}\right)$ given, define operators $\Phi_{1}$ and $\Gamma_{1}$ as follows.

$\Phi_{1}(V): C \rightarrow \mathfrak{L}\left(C^{\nu}([0,1])\right)$, the Banach space of bounded linear maps from $C^{\nu}([0,1])$ to itself, is defined by the following.

For $v \in C^{v}([0,1])$, let $u=\Phi_{1}(V) v$ be the solution of the problem

$$
\begin{gathered}
-u^{\prime \prime}+\left(\frac{\alpha}{d}\left(S\left(u_{0}\right) u_{0}^{\prime} u\right)^{\prime}+P u=v, \quad 0<x<1,\right. \\
d u^{\prime}-\alpha S\left(u_{0}\right) u_{0}^{\prime} u=0 \quad \text { at } x=0,1,
\end{gathered}
$$

and define

$$
\Gamma_{1}(V)=d^{-1} v_{1}\left(\rho R\left(v_{0}\right)-v_{1}\right)+P v_{1},
$$

where $P$ is a positive constant such that $d^{-1}\left(\rho R\left(v_{0}\right)-v_{1}\right)+P>0$ for $0 \leq v_{0} \leq 2,0 \leq v_{1} \leq$ $P_{1}$, where $P_{1}=2 \rho R(1) e^{(\alpha / d) \int_{0}^{1} S(y) d y}$. Then system (3.28) can be written as a fixed point equation $U=A(U)$, where $U=A(V)$ is given by

$$
u_{0}=A_{0}(V), \quad u_{1}=A_{1}(V)=\Phi_{1}(V) \circ \Gamma_{1}(V) .
$$

It is easily seen that $\Gamma_{1}$ satisfies,

$$
\Gamma_{1}\left(u_{0}, 0\right)=0 .
$$

Now we prove the following lemmas.

Lemma 3.4. The operator $A(V)=\left(A_{0}(V), A_{1}(V)\right): \bar{\Omega} \rightarrow C$ is a well-defined completely continuous operator, where

$$
\Omega=\left\{\left(v_{0}, v_{1}\right) \in C: 0 \leq v_{0}<2,0 \leq v_{1}<P_{1}\right\}
$$

Moreover, fixed points of $A$ in $C$ are nonnegative solutions of (3.28).

Proof. First we show that, for $V=\left(v_{0}, v_{1}\right) \in \Omega$, (3.30) has a unique nonnegative solution $u_{0} \not \equiv 0$ and $u_{0} \in C^{2+\nu}([0,1])$. In fact, it is easily seen that $\underline{u}=0$ and $\bar{u}=1$ is a pair of sub- and supersolutions of (3.30). Therefore (3.30) has a solution $u_{0}(x)$ satisfying $0 \leq$ $u_{0}(x) \leq 1$. From the boundary conditions, we have $u_{0} \neq \equiv 0$. From the regularity theory of elliptic equations and the smoothness of $R$, we have $u_{0} \in C^{2+\nu}([0,1])$. Now we prove the 
uniqueness. If there is another solution $u^{0}(x)$ of (3.30). Let $u=u^{0}-u_{0}$, then $u$ satisfies

$$
\begin{gathered}
-u^{\prime \prime}+\mu^{-1} R^{\prime}(\tilde{u}) u v_{1}=0, \quad 0<x<1, \\
u^{\prime}(0)=0, \quad u^{\prime}(1)+\gamma u(1)=0 .
\end{gathered}
$$

From maximum principle, we have $u \equiv 0$.

With $u_{0}$ given, $u_{1}$ is the solution of the problem

$$
\begin{gathered}
-\left(u_{1}^{\prime}-\frac{\alpha}{d} S\left(u_{0}\right) u_{0}^{\prime} u_{1}\right)^{\prime}+P u_{1}=d^{-1} v_{1}\left(\rho R\left(v_{0}\right)-v_{1}\right)+P v_{1}, \quad 0<x<1, \\
d u_{1}^{\prime}-\alpha S\left(u_{0}\right) u_{0}^{\prime} u_{1}=0 \quad \text { at } x=0,1 .
\end{gathered}
$$

Let $G(\tau)=\int_{0}^{\tau} S(y) d y \geq 0$ and $z=e^{-(\alpha / d) G\left(u_{0}\right)} u_{1}$, then we have

$$
u_{1}^{\prime}-\frac{\alpha}{d} S\left(u_{0}\right) u_{0}^{\prime} u_{1}=e^{(\alpha / d) G\left(u_{0}\right)} z^{\prime}
$$

and $z$ satisfies

$$
\begin{gathered}
-\left(e^{(\alpha / d) G\left(u_{0}\right)} z^{\prime}\right)^{\prime}+P e^{(\alpha / d) G\left(u_{0}\right)} z=d^{-1} v_{1}\left(\rho R\left(v_{0}\right)-v_{1}\right)+P v_{1}, \quad 0<x<1, \\
z^{\prime}(0)=z^{\prime}(1)=0 .
\end{gathered}
$$

Observe that $d^{-1}\left(\rho R\left(v_{0}\right)-v_{1}\right)+P>0$, from maximum principle, we have $z>0$. Therefore, $u_{1}>0$. From Schauder's theory for elliptic equations, we have $U=A(V) \in$ $C^{2+\nu}([0,1])$. Therefore $A$ is completely continuous. It is easily seen that the fixed points of $A$ in $C$ are nonnegative solutions of (3.28).

Lemma 3.5. There is an $M>0$ such that

$$
\operatorname{deg}\left(I-A, B_{M}, \mathbf{0}\right)=1
$$

where $B_{M}=\left\{U \in C:\|U\|_{E}<M\right\}$.

Proof. Consider $H(\eta, U):[0,1] \times C \rightarrow E$ defined by

$$
H(\eta, U)=A(\eta U)
$$

We use the homotopy invariance property to $H(\eta, U)$. For $\eta \in[0,1], V \in C$,

$$
U^{\eta}=\left(u_{0}, u_{1}\right)=H(\eta, V)=\left(H_{0}(\eta, V), H_{1}(\eta, V)\right)
$$

is given by

$$
\begin{gathered}
u_{0}^{\prime \prime}=\mu^{-1} R\left(u_{0}\right) \eta v_{1}, \quad 0<x<1, \\
u_{0}^{\prime}(0)=0, \quad u_{0}^{\prime}(1)+\gamma u_{0}(1)=\gamma, \\
-\left(u_{1}^{\prime}-\frac{\alpha}{d} S\left(u_{0}\right) u_{0}^{\prime} u_{1}\right)^{\prime}+P u_{1}=d^{-1} \eta v_{1}\left(\rho R\left(\eta v_{0}\right)-\eta v_{1}\right)+P \eta v_{1}, \quad 0<x<1, \\
d u_{1}^{\prime}-\alpha S\left(u_{0}\right) u_{0}^{\prime} u_{1}=0 \quad \text { at } x=0,1 .
\end{gathered}
$$


It is easy to verify that $H(\eta, U)$ is completely continuous and there is a constant $K$ such that for the solution of $U=H(\eta, U)$, that is, $U=A(\eta U)$, we have $\|U\|_{E} \leq K$. Therefore for $M>K, U=A(\eta U)$ has no solution satisfying $\|U\|_{E}=M$. This implies that for $0 \leq$ $\eta \leq 1, \mathbf{0} \notin(I-H(\eta, \bullet))\left(\partial B_{M}\right)$. Therefore, $\operatorname{deg}\left(I-H(\eta, \bullet), B_{M}, \mathbf{0}\right)$ is a constant for $0 \leq \eta \leq 1$. Thus

$$
\operatorname{deg}\left(I-A, B_{M}, \mathbf{0}\right)=\operatorname{deg}\left(I-H(1, \bullet), B_{M}, \mathbf{0}\right)=\operatorname{deg}\left(I-H(0, \bullet), B_{M}, \mathbf{0}\right) .
$$

But $H(0, U)=A(\mathbf{0})$ is a constant map. Therefore $\operatorname{deg}\left(I-H(0, \bullet), B_{M}, \mathbf{0}\right)=1$. That is, $\operatorname{deg}\left(I-A, B_{M}, \mathbf{0}\right)=1$.

Let

$$
\Delta_{\{0\}}=\left\{U=\left(u_{0}, 0\right) \in \Omega: A(U)=U, u_{0}>0\right\}
$$

then we have $\Delta_{\{0\}}=\{(1,0)\}$. For $\left(v_{0}, v_{1}\right) \in C$, we set $T_{1}\left(v_{0}, v_{1}\right)=\Phi_{1}\left(v_{0}, v_{1}\right) \circ \partial_{1} \Gamma_{1}\left(v_{0}, v_{1}\right)$, where $\partial_{1} \Gamma_{1}\left(v_{0}, v_{1}\right)$ is the partial derivative of $\Gamma_{1}\left(v_{0}, v_{1}\right)$ with respect to $v_{1}$. An easy computation shows that the operator $T_{1}((1,0)): C_{1} \rightarrow C_{1}$ is defined by the following for $v \in C_{1}$, $u=T_{1}((1,0)) v$ is the solution of the boundary value problem

$$
\begin{gathered}
-u^{\prime \prime}+P u=\left(d^{-1} \rho R(1)+P\right) v, \quad 0<x<1, \\
u^{\prime}(0)=u^{\prime}(1)=0 .
\end{gathered}
$$

Now we cite the following theorem.

Theorem 3.6 (see [7]). Let $\mathscr{L}(y)=a_{2}(x) y^{\prime \prime}+a_{1}(x) y^{\prime}+a_{0}(x) y$ be a linear differential operator with no singular points in $\left[x_{1}, x_{2}\right]$, and suppose that $f(x)$ is continuous on $\left[x_{1}, x_{2}\right]$. Assume also that $\left(A_{1}, A_{2}\right) \neq(0,0)$ and $\left(B_{1}, B_{2}\right) \neq(0,0)$. Then the BVP

$$
\mathscr{L}(y)=f(x) ; \quad A_{1} y\left(x_{1}\right)+A_{2} y^{\prime}\left(x_{1}\right)=0, \quad B_{1} y\left(x_{2}\right)+B_{2} y^{\prime}\left(x_{2}\right)=0,
$$

has a unique solution if and only if the associated homogeneous problem $\mathscr{L}(y)=0$ with the same boundary conditions has only the trivial solution.

It is easily seen that the homogeneous problem associated with (3.46)

$$
\begin{aligned}
-u^{\prime \prime}+P u & =0, \quad 0<x<1, \\
u^{\prime}(0) & =u^{\prime}(1)=0,
\end{aligned}
$$

has only the trivial solution when $P>0$. Therefore, from the theorem, we know that for any $v \in C_{1}$, (3.46) has unique solution and by maximum principle, we have $u(x)>0$ on $[0,1]$. That is, $T_{1}((1,0))$ is strongly positive. The eigenvalue problem $T_{1}((1,0)) \psi=\lambda \psi$, is equivalent to

$$
\begin{gathered}
-\psi^{\prime \prime}+P \psi=\lambda^{-1}\left(\frac{\rho}{d} R(1)+P\right) \psi, \quad 0<x<1, \\
\psi^{\prime}(0)=\psi^{\prime}(1)=0 .
\end{gathered}
$$


Obviously, 1 is not an eigenvalue of (3.49) corresponding to a positive eigenfunction. In fact, the eigenvalues of (3.49) are

$$
\lambda_{n}=\frac{(\rho / d) R(1)+P}{P-n^{2} \pi^{2}}, \quad n=0,1,2, \ldots,
$$

and the associated eigenfunctions are $\psi_{n}=\cos (n \pi x)$. Therefore we can see that the eigenvalue that corresponds to the positive eigenfunction is $\lambda=((\rho / d) R(1)+P) / P>1$. This implies that the spectral radius of $T_{1}((1,0))$ is greater than 1 and therefore, from $[9$, Theorem 3.1], we have ind $\left(A, \Delta_{\{0\}}\right)=0$. From Lemma 3.5, we know that for some $M>0$, the set of fixed points of $A$ is in $B_{M}$. Therefore, from Lemma 3.5, we have

$$
\operatorname{ind}(A, \Omega)=\operatorname{ind}\left(A, B_{M}\right)=1 \neq 0=\operatorname{ind}\left(A, \Delta_{\{0\}}\right) .
$$

From [9, Theorem 3.1] mentioned above, we know that (3.28) has at least one positive solution. This implies that (3.1) has solutions of the form $(N, b, 0)$ with $N>0$ and $b>0$. Similarly, we know that (3.1) has solutions of the form $(N, 0, B)$ with $N>0$ and $B>0$. Summarizing the analysis above, we have the following theorem.

Theorem 3.7. System (3.1) has solutions of the form $(N, b, 0)$ and $(N, 0, B)$ with $N>0$, $b>0$, and $B>0$.

3.2. Existence of positive solutions. Now we study the existence of positive solutions of (3.1). As before, we write $N, b$, and $B$ as $u_{0}, u_{1}$, and $u_{2}$, respectively, and write system (3.1) in the form of a fixed point equation as follows.

For $v \in(0,1)$, let

$$
\begin{gathered}
E_{i}=\left\{u \in C^{\nu}([0,1])\right\}, \quad i=0,1,2, \\
C_{i}=\left\{u \in C^{\nu}([0,1]): u \geq 0 \text { on }[0,1]\right\}, \quad i=0,1,2, \\
E=E_{0} \oplus E_{1} \oplus E_{2}, \quad C=C_{0} \oplus C_{1} \oplus C_{2} .
\end{gathered}
$$

For $V=\left(v_{0}, v_{1}, v_{2}\right) \in C$, let $u_{0}=A_{0}(V)$ be the solution of

$$
\begin{gathered}
u_{0}^{\prime \prime}=\mu^{-1}\left(R_{1}\left(u_{0}\right) v_{1}+R_{2}\left(u_{0}\right) v_{2}\right), \quad 0<x<1, \\
u_{0}^{\prime}(0)=0, \quad u_{0}^{\prime}(1)+\gamma u_{0}(1)=\gamma .
\end{gathered}
$$

With $u_{0}$ given, define operators $\Phi_{i}$ and $\Gamma_{i}, i=1,2$, as

$$
\begin{gathered}
\Phi_{1}(V)=\left\{-\frac{d^{2} \bullet}{d x^{2}}+\frac{\alpha}{d}\left(S_{1}\left(u_{0}\right) u_{0}^{\prime} \bullet\right)^{\prime}+P \bullet, d \frac{d \bullet}{d x}-\alpha \bullet S_{1}\left(u_{0}\right) u_{0}^{\prime}\right\}^{-1}, \\
\Phi_{2}(V)=\left\{-\frac{d^{2} \bullet}{d x^{2}}+\frac{\beta}{D}\left(S_{2}\left(u_{0}\right) u_{0}^{\prime} \bullet\right)^{\prime}+P \bullet, D \frac{d \bullet}{d x}-\beta \bullet S_{2}\left(u_{0}\right) u_{0}^{\prime}\right\}^{-1}, \\
\Gamma_{1}(V)=d^{-1} v_{1}\left(\rho_{1} R_{1}\left(v_{0}\right)-v_{1}-v_{2}\right)+P v_{1} \\
\Gamma_{2}(V)=D^{-1} v_{2}\left(\rho_{2} R_{2}\left(v_{0}\right)-v_{1}-v_{2}\right)+P v_{2},
\end{gathered}
$$


where $P$ is a positive constant such that $d^{-1}\left(\rho_{1} R_{1}\left(v_{0}\right)-v_{1}-v_{2}\right)+P>0$ and $D^{-1}\left(\rho_{2} R_{2}\left(v_{0}\right)-\right.$ $\left.v_{1}-v_{2}\right)+P>0$ for $0 \leq v_{0} \leq 2,0 \leq v_{1} \leq P_{1}$, and $0 \leq v_{2} \leq P_{2}$, where $P_{1}=2 \rho_{1} R_{1}(1) e^{(\alpha / d) \int_{0}^{1} S_{1}(y) d y}$ and $P_{2}=2 \rho_{2} R_{2}(1) e^{(\beta / D) \int_{0}^{1} S_{2}(y) d y}$. Then system (3.1) can be written as a fixed point equation $U=A(U)$, where $U=A(V)$ is given by

$$
u_{0}=A_{0}(V), \quad u_{i}=A_{i}(V)=\Phi_{i}(V) \circ \Gamma_{i}(V), \quad i=1,2 .
$$

It is easily seen that $\Gamma_{i}$ satisfies

$$
\Gamma_{i}\left(v_{0}, v_{1}, v_{2}\right)=0 \quad \text { if } v_{i}=0
$$

Similar to the proofs of Lemmas 3.4 and 3.5, we can prove the following two lemmas.

Lemma 3.8. The operator $A(V)=\left(A_{0}(V), A_{1}(V), A_{2}(V)\right): \bar{\Omega} \rightarrow C$ is a well-defined completely continuous operator, where

$$
\Omega=\left\{\left(v_{0}, v_{1}, v_{2}\right) \in C: 0 \leq v_{0}<2,0 \leq v_{1}<P_{1}, 0 \leq v_{2}<P_{2}\right\} .
$$

Moreover, fixed points of $A$ in $C$ are nonnegative solutions of (3.1).

Lemma 3.9. There is an $M>0$ such that

$$
\operatorname{deg}\left(I-A, B_{M}, \mathbf{0}\right)=1
$$

where $B_{M}=\left\{U \in C:\|U\|_{E}<M\right\}$.

For $i=1,2$, let

$$
\Delta_{\{0, i\}}=\left\{U=\left(u_{0}, u_{1}, u_{2}\right) \in \Omega: A(U)=U, u_{0}>0, u_{i}>0, u_{j}=0 \text { for } j \neq i \text { or } 0\right\} .
$$

From the analysis in Section 3.1, we know that $\Delta_{\{0,1\}}$ and $\Delta_{\{0,2\}}$ are nonempty. We consider the following two conditions.

$\left(\mathscr{A}_{1}\right)$ For any $U=\left(\breve{u}_{0}, \breve{u}_{1}, 0\right) \in \Delta_{\{0,1\}}$, the largest eigenvalue of the eigenvalue problem

$$
\begin{gathered}
-\phi^{\prime \prime}+\frac{\beta}{D}\left(S_{2}\left(\check{u}_{0}\right) \check{u}_{0}^{\prime} \phi\right)^{\prime}+P \phi=\lambda^{-1}\left(D^{-1}\left(\rho_{2} R_{2}\left(\check{u}_{0}\right)-\check{u}_{1}\right)+P\right) \phi, \quad 0<x<1, \\
D \phi^{\prime}-\beta S_{2}\left(\check{u}_{0}\right) \check{u}_{0}^{\prime} \phi=0 \quad \text { at } x=0,1,
\end{gathered}
$$

is greater than 1 and for any $U=\left(\hat{u}_{0}, 0, \hat{u}_{2}\right) \in \Delta_{\{0,2\}}$, the largest eigenvalue of the eigenvalue problem

$$
\begin{gathered}
-\psi^{\prime \prime}+\frac{\alpha}{d}\left(S_{1}\left(\hat{u}_{0}\right) \hat{u}_{0}^{\prime} \psi\right)^{\prime}+P \psi=\lambda^{-1}\left(d^{-1}\left(\rho_{1} R_{1}\left(\hat{u}_{0}\right)-\hat{u}_{2}\right)+P\right) \psi, \quad 0<x<1, \\
d \psi^{\prime}-\alpha S_{1}\left(\hat{u}_{0}\right) \hat{u}_{0}^{\prime} \psi=0 \quad \text { at } x=0,1,
\end{gathered}
$$

is greater than 1.

$\left(\mathscr{A}_{2}\right)$ Both eigenvalues of (3.60) and (3.61) are all less than 1.

We have the following theorem.

Theorem 3.10. If either $\left(\mathscr{A}_{1}\right)$ or $\left(\mathscr{A}_{2}\right)$ holds, then (3.1) has at least one positive solution. 
Proof. From Lemmas 3.2 and 3.9, we have ind $(A, \Omega)=1$. Let

$$
\Delta_{\{0\}}=\left\{U=\left(u_{0}, 0,0\right) \in \Omega: A(U)=U, u_{0}>0\right\},
$$

then we have $\Delta_{\{0\}}=\{(1,0,0)\}$ and $\operatorname{ind}\left(A, \Delta_{\{0\}}\right)=0$.

For $i=1,2$, set

$$
T_{i}(V)=\Phi_{i}(V) \circ \partial_{i} \Gamma_{i}(V)
$$

where $\partial_{i} \Gamma_{i}(V)=\partial_{i} \Gamma_{i}\left(v_{0}, v_{1}, v_{2}\right)$ is the partial derivative of $\Gamma_{i}(V)$ with respect to $v_{i}$, then an easy calculation shows that, for $U=\left(\breve{u}_{0}, \breve{u}_{1}, 0\right) \in \Delta_{\{0,1\}}$, the operator $T_{2}\left(\check{u}_{0}, \breve{u}_{1}, 0\right)$ is given by

$$
T_{2}\left(\check{\mathfrak{u}}_{0}, \check{\mathfrak{u}}_{1}, 0\right)=\left(-\frac{d^{2} \bullet}{d x^{2}}+\frac{\beta}{D}\left(S_{2}\left(\check{\mathfrak{u}}_{0}\right) \check{\mathfrak{u}}_{0}^{\prime} \bullet\right)^{\prime}+P \bullet\right)^{-1}\left(D^{-1}\left(\rho_{2} R_{2}\left(\check{\mathfrak{u}}_{0}\right)-\check{\mathfrak{u}}_{1}\right)+P\right)
$$

with the boundary condition in (3.60), and for $U=\left(\hat{u}_{0}, 0, \hat{u}_{2}\right) \in \Delta_{\{0,2\}}$, the operator $T_{1}\left(\hat{u}_{0}, 0, \hat{u}_{2}\right)$ is given by

$$
T_{1}\left(\hat{u}_{0}, 0, \hat{u}_{2}\right)=\left(-\frac{d^{2} \bullet}{d x^{2}}+\frac{\alpha}{d}\left(S_{1}\left(\hat{u}_{0}\right) \hat{u}_{0}^{\prime} \bullet\right)^{\prime}+P \bullet\right)^{-1}\left(d^{-1}\left(\rho_{1} R_{1}\left(\hat{u}_{0}\right)-\hat{u}_{1}\right)+P\right)
$$

with the boundary condition in (3.61). It is easy to verify that $T_{2}\left(\check{\mathcal{u}}_{0}, \check{\mathfrak{u}}_{1}, 0\right)$ and $T_{1}\left(\hat{u}_{0}, 0, \hat{u}_{2}\right)$ are all strongly positive. By using the Krein-Rutman theorem, we can see if $\left(\mathscr{A}_{1}\right)$ holds, then the spectral radii of $T_{2}\left(\breve{u}_{0}, \breve{u}_{1}, 0\right)$ and $T_{1}\left(\hat{u}_{0}, 0, \hat{u}_{2}\right)$ are all less than 1 and $\operatorname{ind}\left(A, \Delta_{\{0,1\}}\right)$ $=1$ and $\operatorname{ind}\left(A, \Delta_{\{0,2\}}\right)=1$. Thus

$$
\text { ind }\left(A, \Delta_{\{0\}}\right)+\operatorname{ind}\left(A, \Delta_{\{0,1\}}\right)+\operatorname{ind}\left(A, \Delta_{\{0,2\}}\right)=0+1+1=2 \neq \operatorname{ind}(A, \Omega)=1 .
$$

From [9, Theorem 3.1], we know that (3.1) has positive solutions.

If $\left(\mathscr{A}_{2}\right)$ holds, the spectral radii of $T_{2}\left(\check{u}_{0}, \breve{u}_{1}, 0\right)$ and $T_{1}\left(\hat{u}_{0}, 0, \hat{u}_{2}\right)$ are all greater than 1 and $\operatorname{ind}\left(A, \Delta_{\{0,1\}}\right)=0$ and $\operatorname{ind}\left(A, \Delta_{\{0,2\}}\right)=0$. Thus

$$
\text { ind }\left(A, \Delta_{\{0\}}\right)+\operatorname{ind}\left(A, \Delta_{\{0,1\}}\right)+\operatorname{ind}\left(A, \Delta_{\{0,2\}}\right)=0+0+0=0 \neq \operatorname{ind}(A, \Omega)=1 .
$$

As before, we conclude that (3.1) has positive solutions.

3.3. Some special cases. Now we consider some special situations.

(I) First we consider $\alpha=0, \beta=0, \rho=1$, and $R_{1}=R_{2}=R$, this is the model discussed in [16], that is,

$$
\begin{gathered}
\mu N^{\prime \prime}-R(N)(b+B)=0, \quad 0<x<1, \\
d b^{\prime \prime}+b(R(N)-b-B)=0, \quad 0<x<1, \\
D B^{\prime \prime}+B(R(N)-b-B)=0, \quad 0<x<1, \\
N^{\prime}(0)=0, \quad N^{\prime}(1)=\gamma(1-N(1)), \\
b^{\prime}(0)=b^{\prime}(1)=0, \\
B^{\prime}(0)=B^{\prime}(1)=0 .
\end{gathered}
$$


In this case, (3.60) and (3.61) become

$$
\begin{aligned}
& -\phi^{\prime \prime}+P \phi=\lambda^{-1}\left(\left(D^{-1}\left(R\left(\check{\mathcal{u}}_{0}\right)-\check{\mathcal{u}}_{1}\right)+P\right) \phi, \quad 0<x<1,\right. \\
& \phi^{\prime}(0)=\phi^{\prime}(1)=0, \\
& -\psi^{\prime \prime}+P \psi=\lambda^{-1}\left(d^{-1}\left(R\left(\hat{u}_{0}\right)-\hat{u}_{2}\right)+P\right) \psi, \quad 0<x<1, \\
& \psi^{\prime}(0)=\psi^{\prime}(1)=0 \text {. }
\end{aligned}
$$

Let

$$
\begin{gathered}
\mathscr{L}_{0}=\left(-\frac{d^{2}}{d x^{2}}\right)^{-1}\left(\frac{R\left(u_{0}\right)-u_{1}}{D}\right), \\
\mathscr{L}_{P}=\left(-\frac{d^{2}}{d x^{2}}+P\right)^{-1}\left(\frac{R\left(u_{0}\right)-u_{1}}{D}+P\right) .
\end{gathered}
$$

We denote the spectral radius of operator $\mathscr{L}$ by $\Upsilon(\mathscr{L})$. It is well known that $\Upsilon\left(\mathscr{L}_{0}\right)>1$ if and only if $\Upsilon\left(\mathscr{L}_{P}\right)>1$ for all $P \geq 0$. Therefore we know that the largest eigenvalues of both (3.69) and (3.70) are greater than 1 if and only if the largest eigenvalues of the following two eigenvalue problems are greater than 1 :

$$
\begin{gathered}
-\phi^{\prime \prime}=\lambda^{-1} D^{-1}\left(R\left(\check{\mathcal{u}}_{0}\right)-\check{\mathcal{u}}_{1}\right) \phi, \quad 0<x<1, \\
\phi^{\prime}(0)=\phi^{\prime}(1)=0, \\
-\psi^{\prime \prime}=\lambda^{-1} d^{-1}\left(R\left(\hat{u}_{0}\right)-\hat{u}_{2}\right) \psi, \quad 0<x<1, \\
\psi^{\prime}(0)=\psi^{\prime}(1)=0 .
\end{gathered}
$$

But it is easily seen that the largest eigenvalue of (3.72) is $\check{\lambda}_{1}=d / D<1$ and the largest eigenvalue of (3.73) is $\hat{\lambda}_{1}=D / d>1$. Therefore neither $\left(\mathscr{A}_{1}\right)$ nor $\left(\mathscr{A}_{2}\right)$ is satisfied. In fact, we can prove that (3.68) has no positive solutions directly. To do this, we first cite the following lemma from [8].

Lemma 3.11. Consider the eigenvalue problem

$$
\begin{gathered}
d \triangle \phi+q(x) \phi=\lambda \phi, \quad x \in \Omega, \\
\frac{\partial \phi}{\partial \nu}=0, \quad x \in \partial \Omega,
\end{gathered}
$$

where $d>0, q(x) \in C^{2+\alpha}(\bar{\Omega})$ for some $\alpha>0$. Let $\lambda_{1}=\lambda(q, d)$ be the unique "principal eigenvalue". Then $\lambda(q, d)$ is a continuous nonincreasing function of $d$, and is strictly decreasing if $q(x)$ is not a constant. Furthermore, the following hold:

(a) $\lambda(q, d) \uparrow Q=\max _{\bar{\Omega}} q(x)$ as $d \rightarrow 0$;

(b) $\lambda(q, d) \downarrow \omega=(1 /|\Omega|) \int_{\Omega} q(x) d x$ as $d \rightarrow \infty$;

(c) if $q_{1}(x) \geq q_{2}(x)$ for $x \in \Omega$, then $\lambda\left(q_{1}, d\right) \geq \lambda\left(q_{2}, d\right)$ with strict inequality if $q_{1}(x) \not \equiv q_{2}(x)$. 
Now we can prove the following theorem.

Theorem 3.12. Equation (3.68) has no positive solution.

Proof. In fact, if (3.68) has a positive solution $U=(N(x), b(x), B(x))$ with $N(x)>$ $0, b(x)>0$, and $B(x)>0$, then $b(x)>0$ and $B(x)>0$ satisfy

$$
\begin{gathered}
d b^{\prime \prime}+(R(N)-b-B) b=0, \quad 0<x<1, \\
D B^{\prime \prime}+(R(N)-b-B) B=0, \quad 0<x<1, \\
b^{\prime}(0)=b^{\prime}(1)=B^{\prime}(0)=B^{\prime}(1)=0 .
\end{gathered}
$$

Because of the positivity of $b(x)$ and $B(x)$, we can consider them as the principal eigenfunctions of the eigenvalue problem

$$
\begin{gathered}
d \phi^{\prime \prime}+q(x) \phi=\lambda \phi, \quad 0<x<1, \\
\phi^{\prime}(0)=\phi^{\prime}(1)=0, \\
B \phi^{\prime \prime}+q(x) \phi=\lambda \phi, \quad 0<x<1, \\
\phi^{\prime}(0)=\phi^{\prime}(1)=0,
\end{gathered}
$$

with $q(x)=R(N(x))-b(x)-B(x)$, associated with the principal eigenvalue $\lambda=$ 0 , respectively. So we have $\lambda(q(x), d)=\lambda(q(x), D)$. Since $q(x)=R(N(x))-b(x)-$ $B(x) \neq$ constant, by Lemma 3.11, this contradicts the assumption $d<D$. The proof of Theorem 3.12 is complete.

(II) Now we consider the following model, which was discussed in [15]:

$$
\begin{gathered}
N^{\prime \prime}-R(N)(b+B)=0, \quad 0<x<1, \\
\left(d b^{\prime}-\alpha b S(N) N^{\prime}\right)^{\prime}+b(\rho R(N)-\theta)=0, \quad 0<x<1, \\
\left(D B^{\prime}-\beta B S(N) N^{\prime}\right)^{\prime}+B(\rho R(N)-\theta)=0, \quad 0<x<1, \\
N^{\prime}(0)=0, \quad N^{\prime}(1)=\gamma(1-N(1)), \\
d b^{\prime}-\alpha b S(N) N^{\prime}=0 \quad \text { at } x=0,1, \\
D B^{\prime}-\beta B S(N) N^{\prime}=0 \quad \text { at } x=0,1,
\end{gathered}
$$

where $\theta>0$ is a constant. In this case, (3.60) and (3.61) become

$$
\begin{gathered}
-\phi^{\prime \prime}+\frac{\beta}{D}\left(S\left(\check{u}_{0}\right) \check{u}_{0}^{\prime} \phi\right)^{\prime}+P \phi=\lambda^{-1}\left(D^{-1}\left(\rho R\left(\check{u}_{0}\right)-\theta\right)+P\right) \phi, \quad 0<x<1, \\
D \phi^{\prime}-\beta S\left(\check{u}_{0}\right) \check{u}_{0}^{\prime} \phi=0 \quad \text { at } x=0,1, \\
-\psi^{\prime \prime}+\frac{\alpha}{d}\left(S\left(\hat{u}_{0}\right) \hat{u}_{0}^{\prime} \psi\right)^{\prime}+P \psi=\lambda^{-1}\left(d^{-1}\left(\rho R\left(\hat{u}_{0}\right)-\theta\right)+P\right) \psi, \quad 0<x<1, \\
d \psi^{\prime}-\alpha S\left(\hat{u}_{0}\right) \hat{u}_{0}^{\prime} \psi=0 \quad \text { at } x=0,1 .
\end{gathered}
$$

The largest eigenvalues of the two eigenvalue problems above are greater than 1 if and only if the largest eigenvalues of the following two eigenvalue problems are 
greater than 1:

$$
\begin{gathered}
-\phi^{\prime \prime}+\frac{\beta}{D}\left(S\left(\check{u}_{0}\right) \check{u}_{0}^{\prime} \phi\right)^{\prime}=\lambda^{-1} D^{-1}\left(\rho R\left(\check{u}_{0}\right)-\theta\right) \phi, \quad 0<x<1, \\
D \phi^{\prime}-\beta S\left(\check{u}_{0}\right) \check{u}_{0}^{\prime} \phi=0 \quad \text { at } x=0,1, \\
-\psi^{\prime \prime}+\frac{\alpha}{d}\left(S\left(\hat{u}_{0}\right) \hat{u}_{0}^{\prime} \psi\right)^{\prime}=\lambda^{-1} d^{-1}\left(\rho R\left(\hat{u}_{0}\right)-\theta\right) \psi, \quad 0<x<1, \\
d \psi^{\prime}-\alpha S\left(\hat{u}_{0}\right) \hat{u}_{0}^{\prime} \psi=0 \quad \text { at } x=0,1 .
\end{gathered}
$$

A special case is $\alpha / d=\beta / D$. For this case, it is easily seen that the largest eigenvalue of (3.81) is $\lambda_{1}=d / D<1$ and the associated eigenfunction is $\phi=\check{u}_{1}$. The largest eigenvalue of (3.82) is $\lambda_{1}=D / d>1$ and the associated eigenfunction is $\psi=\hat{u}_{2}$. Therefore, neither $\left(\mathscr{A}_{1}\right)$ nor $\left(\mathscr{A}_{2}\right)$ is satisfied. In fact, from [15], we know that (3.78) has no positive solutions for this situation.

\section{Acknowledgments}

This work was partly supported by University 2005 Summer Faculty Scholarship of Jackson State University. The author also thanks his program mentor Dr. Xuefeng Wang from Tulane University.

\section{References}

[1] R. A. Adams, Sobolev Spaces, Academic Press, New York, 1975.

[2] N. D. Alikakos, $L^{p}$ bounds of solutions of reaction-diffusion equations, Communications in Partial Differential Equations 4 (1979), no. 8, 827-868.

[3] H. Amann, Dynamic theory of quasilinear parabolic equations. I. Abstract evolution equations, Nonlinear Analysis. Theory, Methods \& Applications 12 (1988), no. 9, 895-919.

[4] - Dynamic theory of quasilinear parabolic systems. III. Global existence, Mathematische Zeitschrift 202 (1989), no. 2, 219-250.

[5] _ Dynamic theory of quasilinear parabolic equations. II. Reaction-diffusion systems, Differential and Integral Equations 3 (1990), no. 1, 13-75.

[6] N. F. Britton, Reaction-Diffusion Equations and Their Applications to Biology, Academic Press, London, 1986.

[7] B. P. Conrad, Differential Equations with Boundary Value Problems, Prentice-Hall, New Jersey, 2003.

[8] J. Dockery, V. Hutson, K. Mischaikow, and M. Pernarowski, The evolution of slow dispersal rates: a reaction diffusion model, Journal of Mathematical Biology 37 (1998), no. 1, 61-83.

[9] L. Dung, Coexistence with chemotaxis, SIAM Journal on Mathematical Analysis 32 (2000), no. 3, 504-521.

[10] D. Henry, Geometric Theory of Semilinear Parabolic Equations, Lecture Notes in Mathematics, vol. 840, Springer, Berlin, 1981.

[11] E. F. Keller and L. A. Segel, Initiation of slime mold aggregation viewed as an instability, Journal of Theoretical Biology 26 (1970), no. 3, 399-415.

[12] K. Osaki and A. Yagi, Finite dimensional attractor for one-dimensional Keller-Segel equations, Funkcialaj Ekvacioj 44 (2001), no. 3, 441-469.

[13] J. Smoller, Shock Waves and Reaction-Diffusion Equations, Fundamental Principles of Mathematical Science, vol. 258, Springer, New York, 1983. 
[14] X. Wang, Qualitative behavior of solutions of chemotactic diffusion systems: effects of motility and chemotaxis and dynamics, SIAM Journal on Mathematical Analysis 31 (2000), no. 3, 535-560.

[15] X. Wang and Y. Wu, Qualitative analysis on a chemotactic diffusion model for two species competing for a limited resource, Quarterly of Applied Mathematics 60 (2002), no. 3, 505-531.

[16] Z. Zhang, Coexistence and stability of solutions for a class of reaction-diffusion systems, Electronic Journal Differential Equations 2005 (2005), no. 137, 1-16.

Zhenbu Zhang: School of Information and Electronics Engineering, Shandong Institute of Business and Technology, Yantai 264005, Shandong, China

Current address: Department of Mathematics, Jackson State University, Jackson, MS 39217, USA

E-mail address: zhenbu.zhang@jsums.edu 


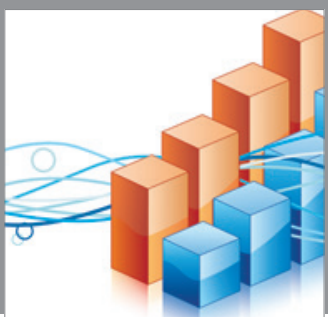

Advances in

Operations Research

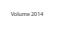

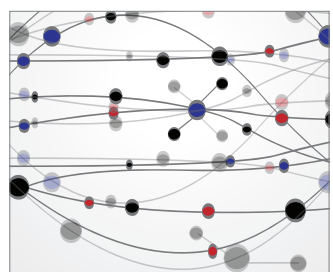

\section{The Scientific} World Journal
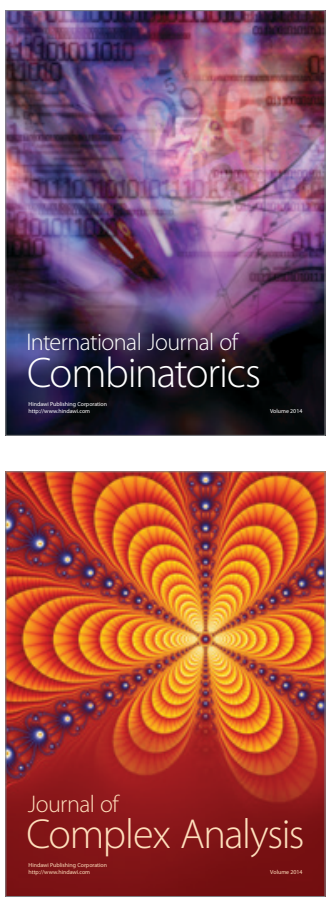

International Journal of

Mathematics and

Mathematical

Sciences
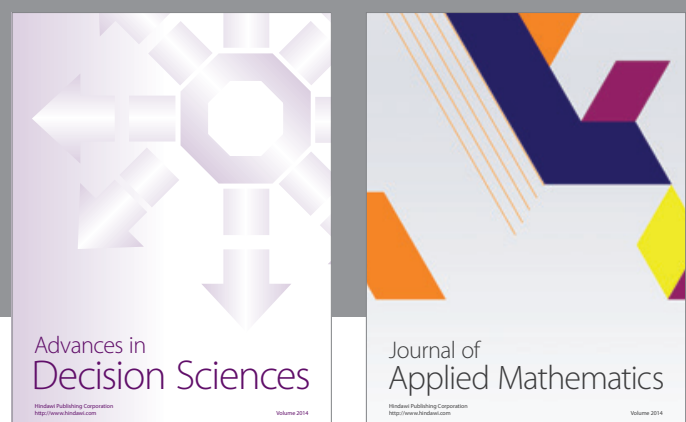

Journal of

Applied Mathematics
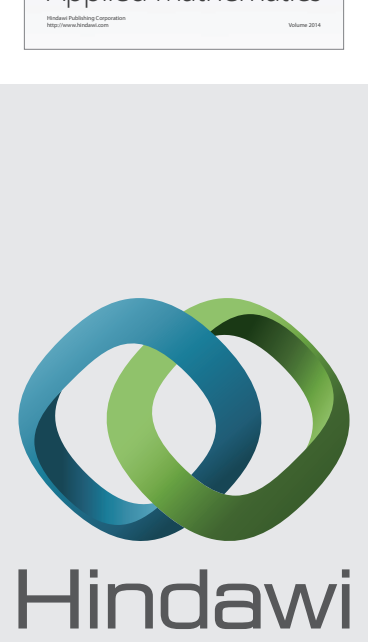

Submit your manuscripts at http://www.hindawi.com
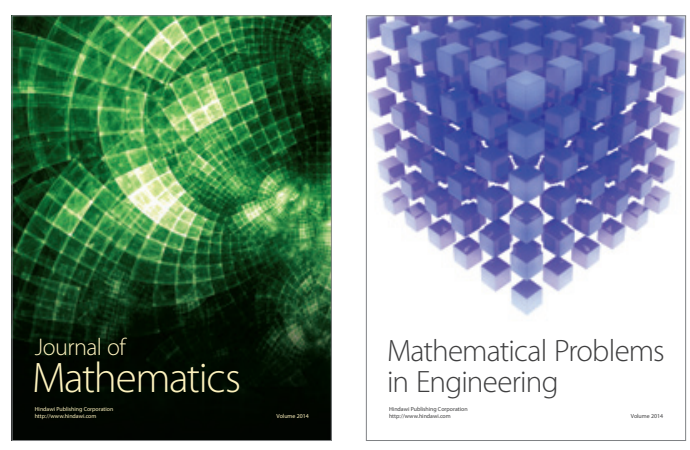

Mathematical Problems in Engineering
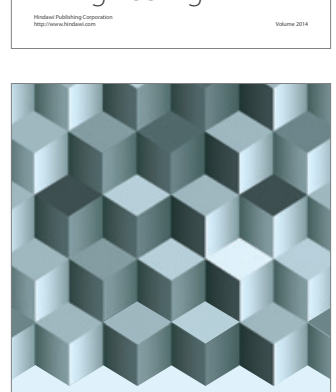

Journal of

Function Spaces
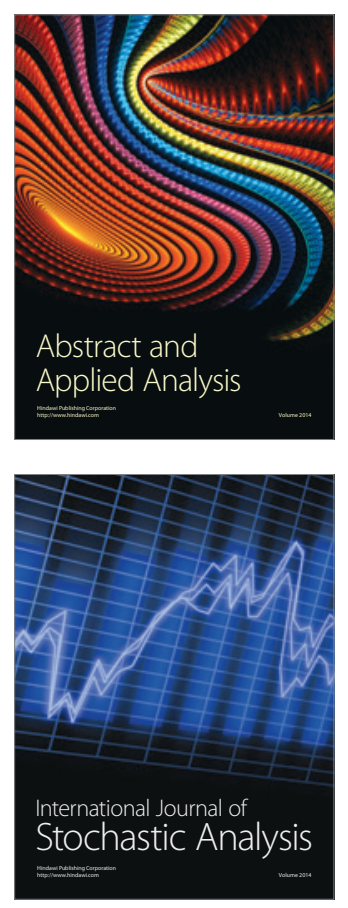

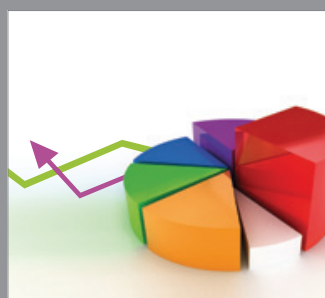

ournal of

Probability and Statistics

Promensencen
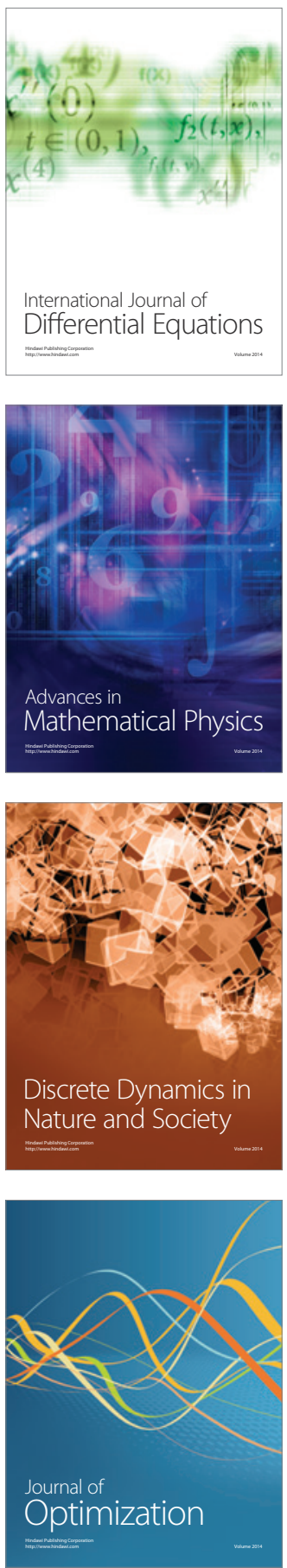